\title{
Les étudiant·e.s en médecine mènent une recherche dans la communauté
}

Rev Med Suisse 2019; 15: 2213-7

Pendant quatre semaines, les étudiant.e.s en médecine de $3^{\mathrm{e}}$ année de l'Université de Lausanne mènent une enquête dans la communauté sur le sujet de leur choix. L'objectif de ce module est de faire découvrir aux futurs médecins les déterminants non biomédicaux de la santé, de la maladie et de l'exercice de la médecine: les styles de vie, les facteurs psychosociaux et culturels, l'environnement, les décisions politiques, les contraintes économiques, les questions éthiques, etc.

Par groupes de 5, les étudiant.e.s commencent par définir une question de recherche originale et en explorent la littérature scientifique. Leur travail de recherche les amène à entrer en contact avec le réseau d'acteurs de la communauté concernés, professionnels ou associations de patients dont ils analysent les rôles et influences respectives. Chaque groupe est accompagné par un·e tuteur.trice, enseignant.e de la Faculté de biologie et de médecine de l'Université de Lausanne. Les étudiant.e.s présentent la synthèse de leurs travaux pendant un congrès de deux jours à la fin du module.

Depuis quelques années, six groupes d'étudiant.e.s ont la possibilité d'effectuer leur travail dans le cadre d'un projet d'immersion communautaire interprofessionnelle à l'étranger, organisé en partenariat avec la Haute école de la santé La Source. Le projet amène les étudiant.e.s à étudier une problématique de santé communautaire dans un pays étranger (Inde et Chine) du point de vue de la médecine et des soins infirmiers. Des groupes de 2 étudiant.e.s en médecine et 2 étudiant.e.s en soins infirmiers sont formés et choisissent un sujet parmi les thématiques proposées par nos partenaires académiques à l'étranger.

Quatre travaux parmi les plus remarquables sont choisis pour être publiés dans la Revue Médicale Suisse.

Module d'immersion communautaire de la Faculté de biologie et de médecine de l'UNIL, sous la direction de:

PR JEAN-BERNARD DAEPPEN (RESPONSABLE), DR JACQUES GAUME (COORDINATEUR), PR PATRICK BODENMANN, PR BERNARD BURNAND, DR AUDE FAUVEL, MME SOPHIE PAROZ, DR DANIEL WIDMER ET PR MADELEINE BAUMANN (HEDS LA SOURCE)

\section{Programmes de promotion de l'activité physique basés sur un système de récompense; effets bénéfiques et effets pervers}

\author{
ELODIE KOLLER, BRUNO MAYOR, ANNA MICHEL, LÉA TRUONG et AURIANA URFER \\ Etudiant.e.s en troisième année de bachelor de la Faculté de biologie et de médecine de l'Université de Lausanne
}

\section{INTRODUCTION}

L'OMS a identifié l'activité physique comme l'une des stratégies prioritaires dans la lutte contre les maladies non transmissibles. ${ }^{1}$ Dans ce contexte, notre intérêt s'est porté plus spécifiquement sur les effets des programmes de prévention de la sédentarité par un programme de promotion de l'activité physique (PPAP) incluant une récompense matérielle (par exemple, des bons cadeaux si le participant atteint son objectif). La littérature met en évidence des problèmes éthiques liés aux programmes de prévention de la sédentarité, comme le risque de stigmatisation $^{2}$ et d'exclusion des personnes vulnérables, ${ }^{3}$ mais non spécifiques à la promotion de l'activité physique. De plus, l'efficacité des PPAP incluant une récompense matérielle n'a été établie qu'à court terme. ${ }^{4,5}$ C'est pourquoi, nous nous sommes demandé dans quelle mesure les PPAP incluant une récompense étaient susceptibles d'avoir des effets négatifs chez certaines personnes.

\section{MÉTHODE}

Nous avons mené des entretiens semistructurés avec différents professionnels de la santé (1 médecin et 1 psychologue du sport, 1 psychiatre, 1 chercheur), 3 représentants de PPAP et des entraîneurs de sportifs amateurs. Ces entretiens ont été soumis à une analyse de contenu.

\section{RÉSULTATS}

Différents facteurs déterminent la participation d'un individu à un PPAP avec récompense matérielle:

1. Des facteurs liés aux programmes eux-mêmes (tous les PPAP étudiés avaient des limitations d'accès).

2. Les objectifs fixés et le mode d'activité physique (ils doivent facilement s'intégrer dans la vie quotidienne et présenter un bénéfice secondaire, par exemple, réduction du temps de trajet, pas d'achat de matériel pour participer).

3. Des facteurs socioculturels (les interviewés expriment que les personnes appartenant à un milieu social défavorisé semblent moins souvent prendre part aux programmes).

Ces facteurs ont pour effet que les participants à ce type de programmes sont majoritairement des individus déjà actifs.

Les personnes interrogées estiment que les programmes sont efficaces à court terme. La stigmatisation des individus inactifs ressort par contre comme l'un des effets négatifs de la valorisation de l'activité physique via les PPAP. Les conditions d'accès excluent certains individus, qui en plus de l'absence d'activité physique, ne 
bénéficient pas des possibles récompenses matérielles. Ces programmes sont néanmoins vus comme ayant aussi des effets indirects positifs (parfois recherchés par les développeurs) comme l'augmentation de la cohésion d'équipe ou un impact écologique.

\section{DISCUSSION}

Les personnes interviewées pensent que les PPAP basés sur un système de récompense matérielle sont efficaces pour favoriser l'activité physique à court terme. Par contre, la population qui bénéficierait le plus d'une activité physique ne correspond pas à celle atteinte par les programmes étudiés, ce qui pose la question de l'équité dans l'accès à la prévention de la sédentarité.

Le risque de stigmatisation trouvé dans la littérature ${ }^{2}$ était souligné par les professionnels de la santé interrogés, lesquels proposaient une participation par équipe ou la valorisation de la progression plutôt que de la performance comme solutions pour diminuer ce risque. Les professionnels de la santé et du sport insistaient en outre sur la nécessité d'un encadrement professionnel pour favoriser la reprise du sport afin d'éviter toutes dérives ou blessures. La littérature ${ }^{2}$ et certains professionnels insistent sur la prudence et la réflexion nécessaires lors de l'implémentation de ce type de programmes afin de réduire les effets négatifs de la promotion de l'activité physique. Les développeurs rencontrés, eux, n'avaient en effet que peu ou pas réfléchi aux effets négatifs potentiellement engendrés par leurs programmes. Finalement, il importe de relever que les développeurs de ce type de programme servent aussi leurs propres intérêts, qu'il s'agisse d'impact marketing ou politique.

Remerciements: Nous remercions les personnes ayant contribué à ce travail, notamment notre tutrice, la Dre Bourquin Sachse.

1 OMS. Suivi de la Déclaration politique de la Réunion de haut niveau de l'Assemblée générale sur la prévention et la maîtrise des maladies non transmissibles. EB134,2013;7.

2 Massé R. Stigmatisation sociale et santé publique; les enjeux éthique. Sante Homme 2012;419:9.

3 Toussaint JF. Ethique et prévention. In Hirsch E. Traité de bioéthique I; fondements, principes, repères.

Toulouse; ERES, 2010: 413-7.

4 Mitchell MS, Goodman JM, Alter DA, et al. Financial Incentives for Exercise adherence in Adults Systematic Review and Meta-analysis. Am J Prev Med 2013;45:65867.

5 Hardman CA, Horne PJ, Lowe CF. Effects of rewards, peer-modelling and pedometer targets on children's physical activity; a school-based intervention study. Psychol Health 2011;263-21.

\title{
Nouveaux e-outils médicaux pour le self-monitoring; les patients enfin acteurs de leur propre santé ?
}

\author{
ANTOINE CHAPOT, ATHITHTHAN KANTHASAMY, GAUTIER LE GAVRIAN, CLÉMENT NUSSBAUMER et RAMA SULTAN \\ Etudiant.e.s en troisième année de bachelor de la Faculté de biologie et de médecine de l’Université de Lausanne
}

\section{INTRODUCTION}

Les maladies chroniques ont un impact non négligeable sur la mortalité et la morbidité globale en Suisse, mais également sur la qualité de la vie comme le démontre une récente étude de l'Office fédéral de la statistique. ${ }^{1}$ Dans ce contexte, les nouvelles technologies médicales trouvent une place grandissante. En effet, le patient commence à mesurer lui-même certains de ses paramètres à l'aide d'outils certifiés comme les tensiomètres ou les lecteurs de glycémie: c'est ce qu'on appelle le self-monitoring. Cette pratique induit la production de données médicales permettant de mieux gérer la maladie. Nous nous sommes donc intéressés à la perception des risques et des bénéfices liés au self-monitoring des patients atteints de maladies chroniques. La littérature traitant essentiellement des impacts clinique et économique, nos recherches se sont dirigées sur l'aspect de mise en pratique dans la vie quotidienne de ces différents outils.

\section{MÉTHODOLOGIE}

Une recherche de littérature scien- tifique a été complétée par une étude qualitative sous forme d'entretiens semistructurés avec différents acteurs du système de santé pour explorer l'aspect communautaire de la problématique: un diabétologue, une infirmière en diabétologie, un néphrologue, un professeur en droit de la santé, un représentant de l'association Diabète Vaud, un médecin spécialiste en e-santé, un médecin conseil d'assurance et un généraliste. Un questionnaire a également été soumis par e-mail à une assurance. Les entretiens ont été basés sur un guide d'entretien général articulé autour de 5 perspectives: le patient, le médecin, l'économie, les aspects juridiques et les perspectives futures. Les interviews ont été enregistrés, analysés et synthétisés par un groupe constitué de deux à trois personnes.

\section{RÉSULTATS}

Les soignants interrogés étaient unanimes sur le fait que le self-monitoring améliore la prise en charge de la maladie. Le patient s'implique plus dans son traitement et l'alliance thérapeutique est ainsi consolidée. Le self-monitoring amène des résultats plus fiables (valeurs de la vie quotidienne) et plus nombreux, ce qui améliore le suivi. «Par rapport aux contrôles faits chez le médecin, ces outils nous permettent d'avoir un plus grand nombre de données mais surtout des mesures qui proviennent du quotidien du patient» (médecin spécialiste en e-santé). La participation du médecin dans l'interprétation des données reste indispensable. L'équipe soignante joue également un rôle important dans l'éducation du patient quant à l'utilisation de ces e-outils et pour le suivi à long terme en donnant des feedbacks. De manière générale, les données amènent une vue plus représentative de la maladie conduisant à une meilleure gestion de la médication par les médecins, diminuant ainsi en grande partie les risques liés à la surmédicalisation. Les soignants ne doivent cependant pas oublier l'impact psychologique que ces outils peuvent générer (par exemple, angoisse de respecter des valeurs seuils). L'utilisation de ces e-outils requiert un bon apprentissage sur leur fonctionnement ainsi que sur l'interprétation des résultats par le malade. Une relation de confiance entre les deux inter- 
locuteurs est d'autant plus importante pour obtenir un bénéfice net dans la prise en charge. D'un point de vue économique, la plupart des outils sont remboursés par l'assurance de base. Sur le long terme, un tel suivi présente des bénéfices pour le patient et pour le système de santé via une baisse des dépenses collatérales (consultations, complications). ${ }^{2,3}$ Aujourd'hui la majorité des données sont rapportées par le patient lors de la consultation et sont donc soumises aux mêmes normes de confidentialité que les autres données médicales (secret médical).

\section{DISCUSSION}

Les e-outils médicaux (tensiomètres, glucomètres) sont des dispositifs présentant des avantages avérés dans les différentes perspectives explorées. Ce constat se traduit par une diminution de la mortalité et de la morbidité selon le contexte dans lequel s'inscrit la maladie (niveau d'éducation, facilité d'accès aux infrastructures hospitalières, etc.). ${ }^{4,5}$ Cependant, il ne faut pas oublier les possibles répercussions psychologiques sur le patient, ce qui nécessite un suivi à long terme. Un travail multidisciplinaire intégrant l'avis de plusieurs branches serait souhaitable (questions éthiques, politiques, etc.). Un suivi médical permettrait également d'éviter des dépenses inutiles découlant d'une mauvaise utilisation de ces outils. D'un point de vue juridique, les risques observés aujourd'hui sur le détournement de données générées restent mineurs. Les bénéfices de ce type de prise en charge étant reconnus, une meilleure formation des médecins de premier recours pourrait se révéler utile.
Remerciements: Nous aimerions remercier notre tutrice Valérie D’Acremont, ainsi que tous les interlocuteurs qui ont accepté de prendre de leur temps pour répondre à nos questions.
1 Office fédéral de la statistique. Principales causes de décès selon le groupe d’âge en 2015. Berne; Office fédéral de la statistique, 2017 Nov (consulté le 2018 Oct 25). Disponible sur; , www.bfs.admin.ch/bfs/fr/home/ statistiques/sante/etat-sante/mortalite-causes-deces/ specifiques.assetdetail.3742891.html

2 Jacob V, et al. Economics of self-measured blood pressure monitoring; a community guide systematic review. Am J Prev Med 2017;53:e105-13.

3 Tucker KL, Sheppard JP, Stevens R, et al. Self-Monitoring of blood pressure in hypertension; A systematic review and individual patient data meta-analysis. PLoS Med 2017;14:e1002389.

4 Martin S, Schneider B, Heinemann L, et al. Self-monitoring of blood glucose in type 2 diabetes and long-term outcome: an epidemiological cohort study. Diabetologia 2006;49: 271-8.

5 McCartney DE, McManus RJ. Self-monitoring and self-management: new interventions to improve blood pressure control. Curr Opin Nephrol Hypertens 2016:25:502-7.

\title{
Suicide chez les adolescents: quelles sont les méthodes actuelles de prévention dans les écoles?
}

\author{
CLAIRE BELLÉGO, MAYARA BOLAY, FLORIANE DAUVIN, ELISA LASSAGNE et MARILOU SIGG \\ Etudiant·e.s en troisième année de bachelor de la Faculté de biologie et de médecine de l’Université de Lausanne
}

\section{INTRODUCTION}

En Suisse, le suicide représentait en 2015 la première cause de mortalité chez les 15-29 ans. ${ }^{1}$ Ces décès, évitables, ont des répercussions sociales, psychologiques et économiques importantes et constituent un problème de santé publique majeur. ${ }^{2}$ Le comportement suicidaire découle généralement d'un cumul de facteurs de risque chez une personne avec une certaine vulnérabilité. ${ }^{3}$ Contrairement à la population adulte, dont les facteurs de risque principaux sont les troubles psychiques, les adolescents sont sensibles à des facteurs de stress importants - conflits relationnels, questions identitaires, manque de ressources sociales. ${ }^{3}$ De plus, l'adolescence étant caractérisée par une forte impulsivité, le risque de passage à l'acte est plus important.

L'objectif de notre recherche était de présenter les méthodes de prévention actuelles du suicide chez les jeunes en milieu scolaire en Suisse romande et la perception qu'en ont ses acteurs.

\section{MÉTHODE}

Nous avons consulté la littérature grise et scientifique et effectué des entretiens semi-structurés avec une psychologue cadre dans une unité dédiée aux adolescents, un membre du Groupe romand prévention suicide (GRPS), deux représentants de l'Association Stop Suicide, une historienne de l'Institut des humanités en médecine du CHUV, trois membres de l'Unité PSPS (Promotion de la santé et prévention en milieu scolaire), dont la coordinatrice du projet GRAFIC (Gestion des ressources d'accompagnement et de formation en cas d'incident critique).

Avec l'accord des intervenants, nous avons enregistré puis retranscrit la plupart des entretiens afin d'en tirer les informations en lien avec notre question de recherche.

\section{RÉSULTATS}

Les actions concrètes pour la prévention du suicide en Suisse romande comprennent principalement des ateliers à destination des jeunes, la distribution de documentation dans les classes, des formations pour les encadrants, des projets d'amélioration du climat scolaire et de la «postvention», c'est-à-dire la prévention contre l'effet de contagion du suicide (tableau 1). Les ateliers visent à développer les stratégies de «coping», processus qui consiste à faire face aux problèmes malgré les émotions qu'ils peuvent susciter, et à promouvoir l'acquisition de réflexes comme informer des personnes ressources. Les intervenants de l'unité PSPS observent un lien net entre l'amélioration du climat scolaire grâce à leurs actions et la 


\section{TABLEAU 1 Méthodes de prévention du suicide chez les adolescents en Suisse romande}

FBM; faculté de biologie et médecine; GRAFIC; Gestion des ressources d’accompagnement et de formation en cas d'incident critique; GRPS; Groupe romand prévention Suicide; PSPS; Promotion de la santé et prévention en milieu scolaire.

Cantons; GE; Genève; VD; Vaud; NE; Neuchâtel; VS; Valais

\begin{tabular}{|c|c|c|c|}
\hline Méthodes & Objectifs & Organisations & Cantons concernés \\
\hline $\begin{array}{l}\text { Ateliers et distribution de documentation } \\
\text { dans les classes }\end{array}$ & $\begin{array}{l}\text { Développer les stratégies de «coping» et adoption } \\
\text { de réflexes bénéfiques }\end{array}$ & Stop Suicide & $\mathrm{GE}, \mathrm{VD}, \mathrm{NE}, \mathrm{VS}$ \\
\hline $\begin{array}{l}\text { Formation des professionnels en contact } \\
\text { avec des adolescents } \\
\text { A. Formation «Faire face au risque suicidaire» } \\
\text { B. Modules de sensibilisation }\end{array}$ & $\begin{array}{l}\text { Etre en mesure de répondre à la demande d’aide et } \\
\text { de réagir face à un jeune avec un comportement } \\
\text { suicidaire }\end{array}$ & $\begin{array}{l}\text { A. GRPS \& FBM de l'Unil, avec le soutien de } \\
\text { Stop Suicide notamment. } \\
\text { B. Stop Suicide, en collaboration avec } \\
\text { Malatavie et le GRPS }\end{array}$ & GE, VD, NE, VS \\
\hline Projets d’amélioration du climat scolaire & $\begin{array}{l}\text { Promouvoir le vivre ensemble et le bien-être des } \\
\text { élèves, diminuant certains facteurs de risque du } \\
\text { suicide (homophobie, harcèlement) }\end{array}$ & Unité PSPS & VD \\
\hline Cellules de crise & $\begin{array}{l}\text { Formation et soutien des cellules de gestion de crise } \\
\text { dans les établissements scolaires afin de prévenir } \\
\text { l'effet de contagion du suicide (postvention) }\end{array}$ & GRAFIC & VD \\
\hline Campagne de sensibilisation (2018) & $\begin{array}{l}\text { Améliorer les connaissances à propos du suicide, } \\
\text { promouvoir bienveillance et soutien }\end{array}$ & $\begin{array}{l}\text { Stop Suicide en collaboration avec Pro } \\
\text { Juventute }\end{array}$ & Echelle nationale \\
\hline
\end{tabular}

diminution des suicides chez les adolescents.

\section{DISCUSSION}

En 2016, l'OFSP, en collaboration avec le GRPS, a établi des objectifs nationaux de prévention du suicide. ${ }^{2}$ Il n'existe cependant pas de directives obligatoires s'appliquant au milieu scolaire, à l'exception de la «postvention» faite par GRAFIC dans le canton de Vaud. Les établissements scolaires sont donc libres d'instaurer les mesures qu'ils jugent adaptées aux besoins de leurs élèves. Selon les membres de l'unité PSPS interrogés, certains établissements sont encore réticents à aborder la question du suicide dans leurs classes jugeant que cette tâche n'incombe pas à l'école ou par crainte de provoquer des idées suicidaires chez certains élèves.

Cette croyance a pourtant été infirmée par l'étude européenne SEYLE (Saving and Empowering Young Lives in Europe $)^{4}$ qui a mis en évidence l'efficacité du programme de prévention du suicide «Youth Aware of Mental health». Celui-ci propose des activités pour appliquer empathie et solidarité afin de reconnaître et réagir face à des problèmes de santé mentale.

Pour conclure, nous n'avons identifié que la postvention comme intervention qui soit rendue obligatoire parmi les cantons romands. Il serait important d'insister sur une prévention systématique pour tous les établissements scolaires, notamment pendant la scolarité obligatoire, puisque cette phase de la vie permet la mise en place des fondements de l'intégrité psychique. ${ }^{3}$
Remerciements: Nous tenons à remercier les différentes personnes qui ont accepté de nous rencontrer, ainsi que notre tutrice, Martine Jacot-Guillarmod, pour son suivi.

1 Stop Suicide le blog de Charlotte Frossard. Le suicide est en hausse chez les jeunes Suisses. Le Temps (En ligne). 26 février 2018 ([cité le 20 juin 2018). Disponible sur; https://blogs.letemps.ch/charlotte-fros-

sard/2018/02/26/le-suicide-est-en-hausse-chez-les-jeunessuisses/

2 Organisation mondiale de la santé. Prévention du suicide; l'état d'urgence mondial. (En ligne). 2014 (cité le 19 juin 2018). Disponible sur; http://apps.who.int/iris/ bitstream/handle/10665/131801/9789242564778_fre.pdf

3 Office fédéral de la santé publique. La prévention du suicide en Suisse: Contexte, mesures à prendre et plan d'action. Rapport sur mandat du Dialogue Politique nationale de la santé. (En ligne). Novembre 2016 (Cité le 19 juin 2018).

4 Wasserman D, Hoven C, Wasserman C, et al. Schoolbased suicide prevention programmes; the SEYLE

cluster-randomised, controlled trial. Lancet 2015;385:1536-44.

\section{Prévention de la consommation d'alcool chez les adolescents à Mangalore}

\section{CLÉMENTINE AUBERT**, BAHIA DIAB*, JOY LUSTER*, BINSHA C. PAPPACHAN*** et MARIE-PAULINE SCHÄUBLIN**}

* Etudiantes en troisième année de bachelor de la Faculté de biologie et de médecine de l'Université de Lausanne; ** Etudiantes en troisième année de bachelor de la Haute école de santé La Source, Lausanne; *** Professeure assistante du Département de psychiatrie du Father Muller College of Nursing, Mangalore

\section{INTRODUCTION}

En Inde, la consommation d'alcool chez les adolescents ${ }^{\text {a }}$ augmente et est devenue un sujet de préoccupation pour le gouvernement. ${ }^{1}$ Une consommation d'alcool précoce accroît le risque de dépen- dance au cours de la vie ${ }^{2}$ avec des répercussions familiales et socio-économiques importantes ${ }^{1}$ et un coût social élevé dans un pays où les ressources dédiées à la prévention sont limitées. ${ }^{3}$ La littérature scientifique se concentre surtout sur les adultes dépendants, délaissant la question des adolescents et des consommateurs non dépendants, qui sont pourtant majoritaires. ${ }^{2}$ Des études indiennes proposent des mesures de prévention primaire, ${ }^{2}$ avec toutefois peu de résultats tangibles. Les objectifs de cette recherche réalisée à Mangalore (Etat du Karnataka, sud-ouest 
de l'Inde) étaient de décrire les mesures de prévention prises contre la consommation d'alcool chez les adolescents et de poser un regard critique sur celles-ci en recueillant le point de vue des professionnels impliqués.

\section{MÉTHODE}

Pour mener cette étude de type qualitatif, 14 entretiens semi-directifs ont été menés auprès d'un échantillon raisonné d'acteurs concernés par la problématique: 3 directeurs d'écoles (privée et publique), 2 psychiatres, 1 représentant des services de protection de l'enfance, 2 travailleurs sociaux, 1 officier de police, 3 étudiantes infirmières, 1 pédiatre et 1 infirmière en pédiatrie. Une analyse de contenu des entretiens, sur un mode inductif, a permis d'élaborer des hypothèses de compréhension dans le but de répondre à notre question de recherche. Le protocole de recherche a reçu l'aval de la commission d'éthique du Father Muller College of Medicine de Mangalore.

\section{RÉSULTATS}

Des mesures de prévention de la consommation d'alcool existent à Mangalore et s'articulent autour de quatre axes: communautaire, éducationnel, médical et légal (tableau 1). Néanmoins, de nombreux facteurs en limitent l'efficacité. Les témoignages relèvent le manque de professionnels spécialisés et les rôles peu délimités entre ceux-ci, compliquant la coordination des actions. Les ressources sont davantage consacrées au traitement de la dépendance qu'à la prévention. De plus, le focus des acteurs de la prévention se porte prioritairement sur les substances illicites telles que le cannabis. Quant à la législation existante limitant l'accès à l'alcool, elle n'est pas toujours respectée.

\section{DISCUSSION}

Dans la société indienne, le rôle de la famille est primordial. Cette dernière est

\begin{tabular}{|c|c|c|c|c|}
\hline & TABLEAU 1 & \multicolumn{2}{|c|}{$\begin{array}{l}\text { Les quatre axes de prévention de la consommation } \\
\text { d'alcool chez les adolescents à Mangalore }\end{array}$} & \\
\hline \multicolumn{3}{|c|}{$\begin{array}{l}\text { Communautaire } \\
\text { - Conseiller scolaire: public et privé, externe ou } \\
\text { interne à l'école } \\
\text { - «Child Helpline»; ligne téléphonique pour les } \\
\text { adolescents des rues } \\
\text { - Travailleur social: si sollicité par les enseignants ou } \\
\text { les parents } \\
\text { - Organisation charitable: pour les adolescents } \\
\text { démunis }\end{array}$} & \multicolumn{2}{|c|}{$\begin{array}{l}\text { Éducationnel } \\
\text { - Famille: figure d'exemple; peut ainsi être un facteur } \\
\text { protecteur ou un facteur de risque de consommation } \\
\text { - Cours «Life Skills»: dispensés dans le cadre de } \\
\text { programmes de prévention en milieu scolaire } \\
\text { - Sensibilisation de l'enseignant à la thématique des } \\
\text { abus de substances } \\
\text { ! La famille est le pilier central de la prévention, } \\
\text { selon tous les intervenants rencontrés }\end{array}$} \\
\hline \multicolumn{3}{|l|}{ Médical } & $\begin{array}{l}\text { Légal } \\
\text { - Vente d'alcool interdite aux moins d } \\
\text { - Taxes sur l'alcool } \\
\text { - Distance minimale entre bar et écol } \\
\text { - Messages publicitaires dissuasifs }\end{array}$ & $\begin{array}{l}18 \text { ans } \\
\text { de } 100 \mathrm{~m}\end{array}$ \\
\hline \multicolumn{3}{|c|}{$\begin{array}{l}\text { ! Le service de pédiatrie n'intervient pas dans la } \\
\text { prévention et la prise en charge des adolescents } \\
\text { consommant de l'alcool; celle-ci étant limitée au } \\
\text { domaine psychiatrique }\end{array}$} & \multicolumn{2}{|c|}{$\begin{array}{l}\text { ! Malgré les messages de prévention dissuasifs, des } \\
\text { slogans publicitaires ciblant les adolescents ren- } \\
\text { forcent le caractère attractif de l'alcool }\end{array}$} \\
\hline
\end{tabular}

l'élément-clé dans la prévention de la consommation d'alcool chez les adolescents et leur prise en charge. ${ }^{4}$ Toutefois, les modèles de prévention ne sont pas toujours adaptés à l'hétérogénéité socio-économique, culturelle et religieuse des familles, ainsi qu'aux différents profils de consommation. La consommation d'alcool dans les familles aisées montre le prestige social alors qu'elle est source de détente chez les familles plus démunies. La stigmatisation sociale et la fierté freinent l'accès à la prévention et aux soins. De nombreuses familles préfèrent affronter seules les problèmes de leur adolescent. ${ }^{4}$ Une sensibilisation à la stigmatisation sociale des adolescents avec un problème d'alcool et de leurs proches mériterait d'être renforcée et adaptée aux différentes communautés.

L'adolescence est une période d'expérimentation où l'individu est facilement influencé par ses pairs, cela constituant une cause d'alcoolisation à cet âge-là. Mangalore est une ville estudiantine, et bon nombre de jeunes y vivent sans leurs parents. La consommation d'alcool des adolescents commence souvent avec cette nouvelle liberté. Une solution serait de réduire l'accessibilité de l'alcool en renforçant les mesures structurelles.

Pour finir, notre étude montre que sur le terrain et ce, contrairement à ce que la littérature laisse penser, la prévention de la consommation d'alcool chez les adolescents ne semble pas réellement être une priorité politique. La situation économique indienne explique peut-être pourquoi les ressources dédiées à la prévention sont plutôt dirigées vers la lutte contre les maladies transmissibles, au détriment de la prévention de la consommation d'alcool. ${ }^{3}$

Remerciements: Nous tenons à remercier le Pr Blaise Guinchard, la Dre Aude Fauvel, les Father Muller Charitable Institutions, ainsi que tous les intervenants rencontrés.

a Selon la définition de l'adolescence de l'OMS qui comprend la tranche d'âge de 10 à 19 ans.

1 National Commission for Protection of Child Rights. Assessment of pattern, profile and correlates of substance use among children in India. 2013. Cité le 20 juin 2018; disponible sur : www.ncpcr.gov.in/view_file. php?fid $=17$

2 Narayana M, Sahoo S, Christoday RJ, Pratima M, Mohan K. Prevention of alcohol dependance: strategies for selective, indicated, and universal prevention. Subst Abus 2011; 32:135-42

3 Benegal V, Velayudhan A, Jain S. Social costs of alcoholism; a Karnataka perspective. NIMHANS J 2000;18:1-12.

4 Sunder L, Adarsh, Pankaj. Textbook of community medicine: preventive and social medicine. 4 th ed. New Delhi; CBS publisher \& distributors; 2014;161-626. 